\title{
Lung Ultrasound in Assessment of Patients with COVID-19
}

\author{
Jianjian Liu, MD ${ }^{a, 1}$, Xin Ma, MD ${ }^{a, 1}$, Xujuan Pu, MD ${ }^{a}$, Jingjing Huang, MD ${ }^{a}$, Zongguo Yang, MD ${ }^{a}$, Xia Shi, MD ${ }^{a}$, \\ Dou Wu, MD ${ }^{a}$, Liping Sun, MD ${ }^{b, *}$, Hui Zhang, MD ${ }^{a, *}$ \\ ${ }^{a}$ Department of Medical Ultrasound, Shanghai Public Health Clinical Center, Fudan University, Shanghai, China; ${ }^{b}$ Department of \\ Medical Ultrasound, Shanghai Tenth People's Hospital, Ultrasound Research and Education Institute, Tongji University Cancer Center, \\ Shanghai Engineering Research Center of Ultrasound Diagnosis and Treatment, Tongji University School of Medicine, Shanghai, China \\ Received April 14, 2020; revision received April 19, 2020; accepted April 20, 2020
}

Objective: To explore the diagnostic value of pulmonary ultrasound in patients with coronavirus disease 2019 (COVID-19).

Methods: A total of 27 COVID-19 patients (mean age \pm standard deviation, $62.6 \pm 12.4$ years) were enrolled in this study, including 14 males (51.9\%) and 13 females (48.1\%). Clinical information of these patients was collected, including age, gender, clinical manifestations, medical history, and laboratory indicators. All patients underwent ultrasound examination of their lungs and the results were analyzed.

Results: Ultrasound examination of the lungs showed that the lesions in 24 patients were located in both lungs (88.9\%), 1 patient's lesions were located in the left lung (3.7\%), and 2 patients' lesions were located in the right lung (7.4\%). Patients with abnormal lung changes mainly had consolidation, air bronchogram, pisces sign, comet-tail artifact, pleural effusion, fusion B-line, and either multiple or few B-lines.

Conclusion: Pulmonary ultrasound examination was a convenient and radiation-free examination method, which could have important clinical value in evaluation of patients with COVID-19.

Key words: Lung ultrasound; COVID-19; Novel coronavirus; Pneumonia

Advanced Ultrasound in Diagnosis and Therapy 2020;02:112-118

DOI: $10.37015 / A U D T .2020 .200039$

$P^{1}$ oronavirus disease 2019 (COVID-19) is an acute infectious disease caused by a novel coronavirus previously unknown in humans before, SARSCoV-2 [1]. The initial symptoms of the patients are mostly fever, fatigue, and dry cough, with the gradual appearance of breathing difficulties and other serious manifestations [2]. Most patients have a good prognosis, although some severe cases may develop acute respiratory distress syndrome or septic shock, or even death. At present, there has been no effective antiviral drugs against the pathogen, and treatment has primarily involved isolation and symptomatic alleviation [3].

At present, the imaging evidence of this disease is mainly based on high-resolution computed tomography (HRCT). Pulmonary ultrasound has been used in clinical practice in the differentiation and diagnosis of pulmonary effusion lesions such as pleural effusion, pneumothorax, as well as in evaluation of dyspnea and acute respiratory failure. It has also been used in the guidance and monitoring of treatment [4]. As a rapid, radiation-free and convenient method for the visual examination of lung diseases, pulmonary ultrasound plays a certain role in the

\footnotetext{
${ }^{1}$ Co-first authors

*Corresponding Authors: Hui Zhang, MD, Departments of Medical Ultrasound, Shanghai Public Health Clinical Center, Fudan University, No. 2501 Caolang Road, Jinshan District, Shanghai 201508, China. e-mail: zhanghui@shphc.org.cn; Liping Sun, MD, Department of Medical Ultrasound, Shanghai Tenth People's Hospital, Ultrasound Research and Education Institute, Tongji University Cancer Center, Shanghai Engineering Research Center of Ultrasound Diagnosis and Treatment, Tongji University School of Medicine, 301 Yanchang Middle Road, Shanghai 200072, China.e-mail: sunliping_s@126.com
}

2576-2508/O AUDT $2020 \cdot \mathrm{http}: / / w w w . A U D T . o r g$

This is an open-access article distributed under the terms of the Creative Commons Attribution 4.0 International license, which permits unrestricted use, distribution and reproduction in any medium provided that the original work is properly attributed. 
diagnosis and treatment of critically ill patients, including COVID-19 [5]. The lungs are the main gas-bearing organ in human body. This makes ultrasonography in normal lung tissue difficult since propagation of ultrasound in the presence of gas leads to total reflection. However, in pathological conditions, the gas to liquid ratio within the lung tissue will change, which can appear on ultrasound as artifacts and abnormal signs. Lung ultrasonography mainly includes 10 basic signs: normal lung signs (bat sign, pleural sliding sign, A-line), pleural effusion signs (quadrilateral sign, sine wave sign), lung consolidation signs (debris sign, tissue like sign), interstitial syndrome (lung rocket sign) and pneumothorax manifestations (stratospheric sign, lung point) [6,7]. In recent years, with the continuous development of ultrasonic technology, ultrasonic diagnosis of pulmonary diseases has become an important means of examination and monitoring treatment effect in the world, and the visualization of pulmonary pathophysiology has been realized, which is known as the visual "stethoscope" [8].In this study, our aim was to explore the diagnostic value of pulmonary ultrasound in COVID-19 patients.

\section{Materials and Methods}

\section{Patients}

This study was approved by the ethics committee of Shanghai Public Health Clinical Center. A total of 27 COVID-19 patients were enrolled in this study. Suspected cases were also classified as confirmed cases if they had one of the following evidences: (1) novel coronavirus nucleic acid tested positive; (2) the gene sequencing of the virus was highly homologous to the virus; (3) antibodies corresponding to novel coronavirus were present in the serum [9]. All of the confirmed patients had their lungs examined by ultrasound.

Clinical information of patients was collected, including age, gender, clinical manifestations, medical history, and so on. In addition, laboratory examination indicators of the patients were collected, including $\mathrm{T}$ cell count, routine blood examination results, liver function tests, renal function indicators, and coagulation indicators.

\section{Pulmonary ultrasonography}

An ultrasound machine (Resona 8, Mindray Medical, Shenzhen, China) with abdominal convex array probe was used in this experiment. Ultrasound examination methods and procedures are in accordance with Bedside Lung Ultrasound in Emergency protocol (BLUE). Patients were placed in supine and lateral positions (supine to examine the upper and lower BLUE points; lateral position to examine the PLAPS, diaphragm, and posterior BLUE points). The upper BLUE point is located between the third and fourth metacarpal knuckles of the upper hand, the lower BLUE point is located between the lower palm of the hand, and the lower BLUE point is located between the lower scapula and the spine. The lower border of the lower little finger of the hand is the diaphragm line, and the intersection point between the posterior extension line and the midaxillary line is the diaphragm line. The intersection of the lower BLUE point and the posterior axillary line is the PLAPS point.

The probe was placed in the intercostal space of the above 5 standard points, and transverse and longitudinal scanning was conducted at each point. It was advisable to scan the PLAPS points with the probe pointing in the direction of the anterior sternum. At the same time, the projection area on the body surface of the patient suggested by CT can be compared with the corresponding scan, and the markers can coexist in the figure. We marked the images of each point: the upper BLUE point was marked as 1, the lower BLUE point was marked as 2, the diaphragm point was marked as 3, the PLAPS point was marked as 4, the latter BLUE point was marked as 5 , and then left and right marks were made. For example, the top BLUE point on the right was R1.

\section{Pulmonary ultrasonographic features}

The main observed indicators were pleural line, B-lines, consolidation, pleural effusion, pneumothorax, and so on, which were defined as follows. Pleural line was the echo reflection formed by the difference of acoustic impedance between the pleura and the lung surface. Under real-time ultrasound, the visceral pleura and parietal pleura slide horizontally relative to each other with the respiratory movement of lung tissue, which was called the pulmonary sliding sign. B-lines began at the pleural line and were perpendicular to it, radiating longitudinally to the depth of the field. Lung consolidation referred to the manifestation of solid tissue echo after the complete loss of lung tissue gasification, showing variably sized patchy or "liver-like changes", which could be accompanied by bronchopneumatic signs. Pleural effusion presents on ultrasound as hypoechoic or anechoic regions in the thorax, with stripy separation. Attention should be paid to differentiate the pleural effusion from the consolidated lung tissue during empyema.

Measurement of lung consolidation range: lateral scanning image was used to measure the largest left and right meridional and the largest anteroposterior diameter of the lesion, and longitudinal scanning image was used to measure the largest upper and lower meridional 
diameter of the lesion. Pleural effusion was divided into small, medium and large amounts. A small pleural effusion was that with a liquid depth less than $2 \mathrm{~cm}$, and the amount of effusion was less than $500 \mathrm{ml}$; a liquid depth of 2-4 cm was a medium pleural effusion with 500-1000 ml of effusion; and a large pleural effusion had a liquid depth greater than $4 \mathrm{~cm}$ with an effusion amount greater than $1000 \mathrm{ml}$.

\section{Statistical analysis}

Continuous variables (including age, and laboratory data) were expressed as mean \pm standard deviation (SD); and categorical variables (including gender, disease type, ultrasound manifestations, and clinical symptoms) were expressed as numbers and percentage.

\section{Results}

\section{Clinical characteristics}

The demographics of COVID-19 patients in this study are shown in Table 1. The average age of the 27 patients was 62.6 years (SD, 12.4 years), including 14 males $(51.9 \%)$ and 13 females $(48.1 \%)$. The average length of hospitalization was 25 days, with 15 mild cases $(55.6 \%), 11$ severe cases $(40.7 \%), 1$ life-threating case (3.7\%), and 2 patients died (7.4\%). Some patients had other comorbidities, including 10 with hypertension (37.0\%), 2 with diabetes (7.4\%), 2 with cardiovascular disease (7.4\%), and 3 with tumors (11.1\%). The patients in this study underwent different clinical treatment, including 21 patients receiving antiviral drug therapy $(77.8 \%), 15$ patients receiving antibiotic therapy $(55.6 \%)$, 13 patients receiving intravenous immunoglobulin $\gamma$ therapy $(48.1 \%), 13$ patients receiving thymalfasin therapy $(48.1 \%), 9$ patients receiving glucocorticoid therapy $(33.3 \%)$, and 11 patients receiving traditional Chinese medicine treatment (40.7\%).

In the patients' laboratory values, the number of $\mathrm{T}$ cells decreased, including $\mathrm{CD} 3+, \mathrm{CD} 4+$, and $\mathrm{CD} 8+$ cells (446, 267 and 172 cells $/ \mathrm{ml}$, respectively). Patient albumin levels were also slightly lower than normal $(38.3 \pm 3.3 \mathrm{~g} / \mathrm{L})$. Values of hypersensitive C-reactive protein and D-Dimer were higher than normal, $36.3 \pm$ $33.9 \mathrm{mg} / \mathrm{L}$ and $0.77 \mu \mathrm{g} / \mathrm{ml}$, respectively. In liver function tests, the values of glutamate transaminase and lactate dehydrogenase were higher than the normal values, 35.5 \pm 17.1 and $327.9 \pm 127.2 \mathrm{U} / \mathrm{L}$, respectively (Table 2 ).

\section{Chest CT findings of patients}

The chest CT findings of 27 patients with coronavirus 2019-nCov pneumonia were GGO (18, $66.7 \%)$, consolidation $(20,74.1 \%)$, fibrosis $(15,55.6 \%)$, mixed ground glass opacity $(11,40.7 \%)$, pleural effusion
(2/7.4\%), including left lung lesions in 2 cases (7.4\%), right lung lesions in 3 cases (11.1\%), diffuse lung lesions in 22 cases $(81.5 \%)$ ( Table3).

\begin{tabular}{lc} 
Table 1 Baseline features and disease history in 27 COVID-19 patients \\
\hline \multicolumn{1}{|c}{ Variables } & $n=27$ \\
\hline Age, years, mean \pm SD & $62.6 \pm 12.4$ \\
\hline Male, $n(\%)$ & $14(51.9)$ \\
\hline Disease type, $n(\%)$ & $15(55.6)$ \\
\hline Mild & $11(40.7)$ \\
\hline Severe & $1(3.7)$ \\
\hline Life-threating type & $2(7.4)$ \\
\hline Mortality, $n$ (\%) & $25 \pm 7.0$ \\
\hline Hospitalization period, days, mean \pm SD & $29 \pm 6.9$ \\
\hline Course of Disease, days, mean \pm SD & \\
\hline Disease history, $n$ (\%) & $10(37.0)$ \\
\hline Hypertension & $2(7.4)$ \\
\hline Diabetes & $2(7.4)$ \\
\hline Cardiovascular diseases & $3(11.1)$ \\
\hline Tumor & \\
\hline
\end{tabular}

Therapeutic strategy, $n(\%)$

\begin{tabular}{|lc|}
\hline Antivirus drugs & $21(77.8)$ \\
\hline Antibiotics & $15(55.6)$ \\
\hline Intravenous immunoglobulin $\gamma$ & $13(48.1)$ \\
\hline Thymalfasin & $13(48.1)$ \\
\hline Glucocorticoid & $9(33.3)$ \\
\hline Traditional Chinese Medicine & $11(40.7)$ \\
\hline
\end{tabular}

Table 2 Laboratory tests in 27 COVID-19 patients

\begin{tabular}{lc}
\multicolumn{1}{c}{ Variables } & Value \\
\hline $\mathrm{T}$ cells, cells/ml, median (IQR) & \\
$\mathrm{CD}^{+} \mathrm{T}$ cell & $446(469)$ \\
$\mathrm{CD}^{+} \mathrm{T}$ cell & $267(289)$ \\
$\mathrm{CD} 8^{+} \mathrm{T}$ cell & $172(167)$
\end{tabular}

Blood routine tests, mean $\pm \mathrm{SD}$

\begin{tabular}{lr} 
White blood cells, $10^{3} / \mathrm{mm}^{3}$ & $5.8 \pm 2.6$ \\
Red blood cells, $10^{3} / \mathrm{mm}^{3}$ & $4.1 \pm 0.7$ \\
\hline
\end{tabular}




\begin{tabular}{lc}
\hline Platelet, $10^{3} / \mathrm{mm}^{3}$ & $166 \pm 95$ \\
\hline Neutrophils, $10^{3} / \mathrm{mm}^{3}$ & $4.1 \pm 2.0$ \\
\hline Lymphocytes, $10^{3} / \mathrm{mm}^{3}$ & $1.1 \pm 1.0$ \\
\hline Monocytes, $10^{3} / \mathrm{mm}^{3}$ & $0.54 \pm 0.39$ \\
\hline Hypersensitive C-reactive protein, $\mathrm{mg} / \mathrm{L}$ & $36.3 \pm 33.9$ \\
\hline Liver function tests, mean $\pm \mathrm{SD}$ & \\
\hline Alanine aminotransferase, $\mathrm{U} / \mathrm{L}$ & $28.5 \pm 18.7$ \\
\hline Aspartate aminotransferase, $\mathrm{U} / \mathrm{L}$ & $35.5 \pm 17.1$ \\
\hline Lactate dehydrogenase, $\mathrm{U} / \mathrm{L}$ & $327.9 \pm 127.2$ \\
\hline Total bilirubin, $\mu$ mol/L & $10.4 \pm 4.0$ \\
\hline Albumin, $\mathrm{g} / \mathrm{L}$ & $38.3 \pm 3.3$ \\
\hline Globulin, $\mathrm{g} / \mathrm{L}$ & $28.3 \pm 4.0$ \\
\hline Renal function test, mean $\pm \mathrm{SD}$ & $13.4 \pm 0.8$ \\
\hline Urea, mmol/L & $0.77(0.75)$ \\
\hline Creatinine, $\mu$ mol/L & $104 \pm 44$ \\
\hline Serum cystatin $\mathrm{C}, \mathrm{mg} / \mathrm{L}$ & $1.01 \pm 0.07$ \\
\hline Coagulation function tests & \\
\hline International normalized ratio, mean $\pm \mathrm{SD}$ & $6.4 \pm 3.8$ \\
\hline Prothrombin activity, mean $\pm \mathrm{SD}$ & \\
\hline Prothrombin time, second, mean $\pm \mathrm{SD}$ & $\mu \mathrm{g} / \mathrm{ml}, \mathrm{median}(\mathrm{IQR})$ \\
\hline
\end{tabular}

Table 3 Chest CT imaging in 27 COVID-19 patients

\begin{tabular}{|cc}
\hline \multicolumn{1}{c}{ Variables } & Value \\
\hline Chest CT imaging, $n(\%)$ & $18(66.7)$ \\
\hline Ground glass opacity (GGO) & $20(74.1)$ \\
\hline Consolidation & $15(55.6)$ \\
\hline Fibrosis & $11(40.7)$ \\
\hline Mixed ground glass opacity & $2(7.4)$ \\
\hline Pleural effusion & \\
\hline Lesion location, $n$ (\%) & $2(7.4)$ \\
\hline Left lung & $3(11.1)$ \\
\hline Right lung & $22(81.5)$ \\
\hline Bilateral lungs
\end{tabular}

\section{Pulmonary ultrasonographic features}

Ultrasound examination of the lungs showed that the lesions in 24 patients were located in both lungs $(88.9 \%)$, 1 patient's lesions were located in the left lung (3.7\%), and 2 patients' lesions were located in the right lung (7.4\%). Findings in patients with lung changes mainly consisted of consolidation, air bronchogram, pisces sign, comet-tail artifact, pleural effusion, fusion B-line, and either multiple or few B-lines. The specific results are shown in Table 4.

\section{Pulmonary ultrasonographic features comparison with chest $C T$}

We presented pulmonary ultrasonographic features and chest CT manifestations from 5 cases of COVID-19. As shown below, consolidation in

Table 4 Manifestations of lung ultrasound in 27 COVID-19 patients

\begin{tabular}{|c|c|c|c|c|c|c|c|c|}
\hline \multirow{2}{*}{ Manifestations $n(\%)$} & \multicolumn{4}{|c|}{ Right lung } & \multicolumn{4}{|c|}{ Left lung } \\
\hline & $\mathrm{R} 1$ & $\mathrm{R} 2$ & R3 & $\mathrm{R} 4$ & L1 & L2 & L3 & L4 \\
\hline Consolidation & $9(33.3)$ & $13(48.2)$ & $12(44.4)$ & $10(37.0)$ & $4(14.8)$ & $12(44.4)$ & $11(40.7)$ & $8(29.6)$ \\
\hline Air bronchogram & $3(11.1)$ & $4(14.8)$ & $5(18.5)$ & $5(18.5)$ & $0(0)$ & $5(18.5)$ & $3(11.1)$ & $2(7.4)$ \\
\hline Pisces sign & $5(18.5)$ & $3(11.1)$ & $2(7.4)$ & $7(25.9)$ & $2(7.4)$ & $3(11.1)$ & $1(3.7)$ & $6(22.2)$ \\
\hline Comet-tail artifact & $2(7.4)$ & $4(14.8)$ & $1(3.7)$ & $1(3.7)$ & $4(14.8)$ & $2(7.4)$ & $3(11.1)$ & $1(3.7)$ \\
\hline Pleural effusion & $2(7.4)$ & $0(0)$ & $4(14.8)$ & $11(40.7)$ & $0(0)$ & $0(0)$ & $2(7.4)$ & $6(22.2)$ \\
\hline Fusion B-line & $10(37.0)$ & $11(40.7)$ & $11(40.7)$ & $8(29.6)$ & $12(44.4)$ & $8(29.6)$ & $8(29.6)$ & $12(44.4)$ \\
\hline Multiple B-line & $11(40.7)$ & $2(7.4)$ & $5(18.5)$ & $7(25.9)$ & $4(14.8)$ & $7(25.9)$ & $7(25.9)$ & $6(22.2)$ \\
\hline Few B-line & $1(3.7)$ & $6(22.2)$ & $2(7.4)$ & $4(14.8)$ & $2(7.4)$ & $4(14.8)$ & $2(7.4)$ & $0(0)$ \\
\hline Negative & $2(7.4)$ & $0(0)$ & $1(3.7)$ & $0(0)$ & $2(7.4)$ & $1(3.7)$ & $1(3.7)$ & $1(3.7)$ \\
\hline
\end{tabular}


pulmonary ultrasonographic was in line with chest CT manifestations (case 1, 3, 4 and 5). In addition, the liver- like consolidation in pulmonary ultrasonographic was also consistent with that in chest CT scans (case 2).
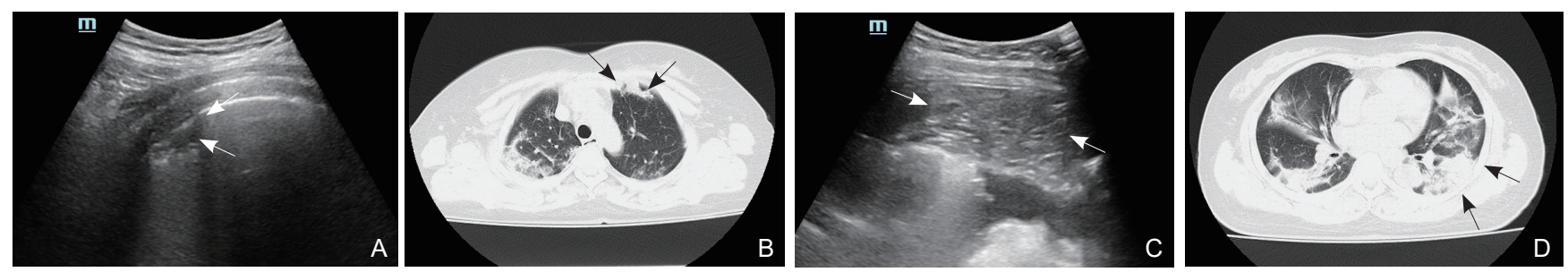

Figure 1 Case 1, a 66 years-old female. (A and C) Ultrasound demonstration of lung consolidation (L1 and L3) is basically consistent with CT examination (B and D).
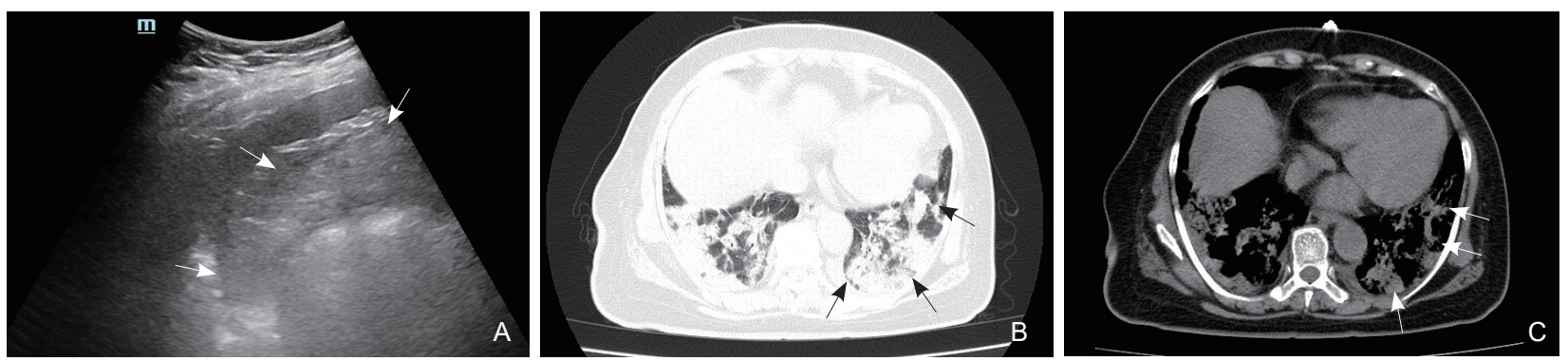

Figure 2 Case 2, a 63 years-old female. Ultrasound observation of lung consolidation in L3 score (A) is basically consistent with CT findings (B and C).
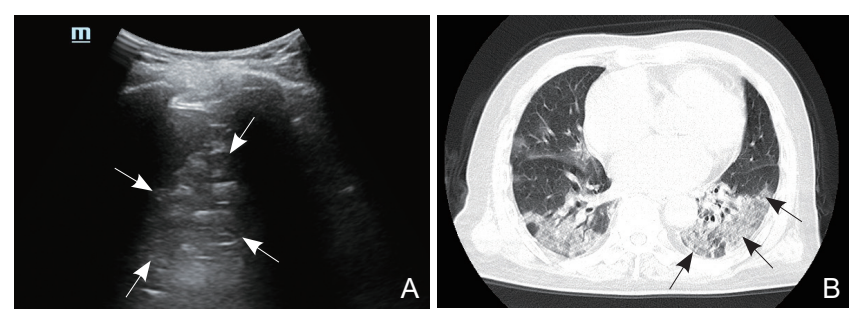

Figure 3 Case 3, a 78 years-old male. Ultrasound visualization of lung consolidation in L3 score (A) is basically consistent with CT (B).

\section{Discussion}

In recent years, lung ultrasound has become a simple and reliable method for the clinical diagnosis of pulmonary diseases. Lung ultrasound can provide a lot of real-time and effective information for the changes of pulmonary morphology [10]. The changes of pulmonary diseases can be determined by indirect signs of ultrasound, such as the presence of pleural abnormalities, the disappearance of A-line, an increase in B-lines, and
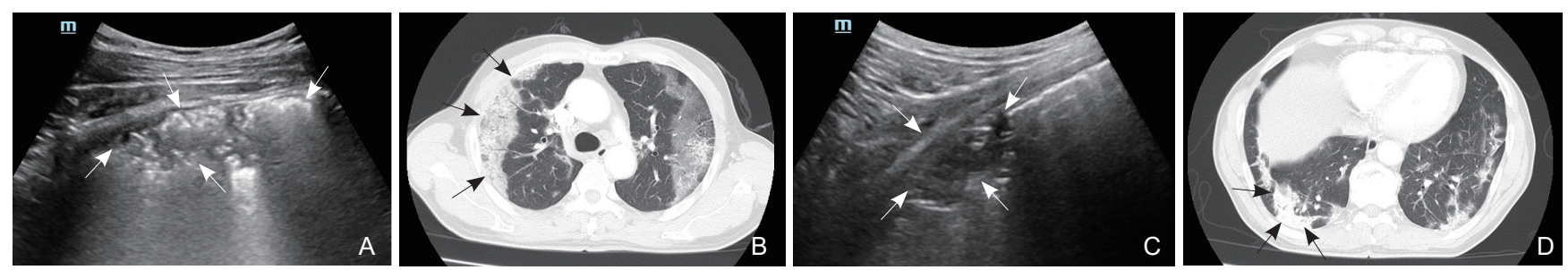

Figure 4 Case 4 of a 65-years-old male. (A and C) Ultrasound observation of lung consolidation (R1, R3) is basically consistent with lung CT results (B and D).
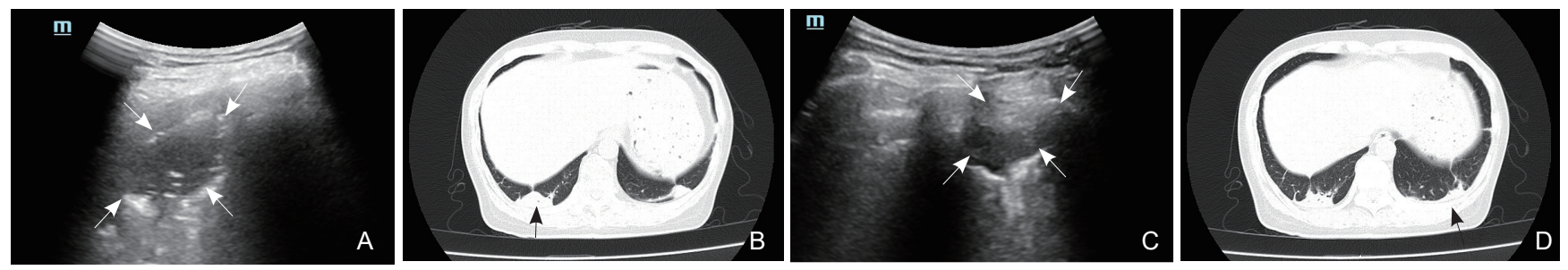

Figure 5 Case 5, a 62-years-old female,. (A and C) Ultrasound observation of lung consolidation (L4, R3) is basically consistent with lung CT findings (B and D). 
lung consolidation. Based on the international consensus of ultrasonic diagnosis for lung diseases published by the World Interactive Network Focused On Critical Ultrasound (WINFOCUS), lung ultrasound can not only accurately diagnose a variety of lung diseases, but has a higher accuracy and sensitivity than chest X-ray for the diagnosis of pneumothorax, lung consolidation, and pleural effusion [4].

Despite air limitations, pulmonary sonography has been shown to be significant in the assessment of a wide range of acute and chronic conditions, from cardiogenic pulmonary edema to acute lung injury, from pneumothorax to pneumonia, from interstitial lung disease to pulmonary infarction and contusion. On the other hand, the technology is simple to learn, which has few technical constraints, and is fast, portable, repeatable, and non-ionizing, making it suitable for use in a variety of situations 11 . The main manifestations of lung ultrasound used to diagnose community-acquired pneumonia in adults are different degrees of lung consolidation and pleural abnormalities, some with fused B-lines and pleural effusion. Among them, pleural abnormalities are mainly manifested in the period of acute inflammation, most of which show the "crushing sign". When the lung tissue produces inflammatory exudation, the presence of B-lines is related to the loss degree of pulmonary ventilation.

Ultrasound evaluation of the lungs can be limited because the ultrasound rapidly fades away in the air, and the lung parenchyma is difficult to image directly because of the loss of echo between the lung and surrounding parenchyma due to the presence of air [12]. In a normally inflated lung, the only tissue that can be detected is the pleura, which appears as a high horizontal line, called the pleura line. However, whether this line is due to the reflection of false image between alveolar air and chest wall soft tissue or the real-time image of pleura is still controversial. When the air content decreases, some exudates, leaks, collagen and blood will increase the density of the lung, and the rebound loss effect between the lung and the surrounding tissues will be reduced, so that the ultrasound can reflect the image of the deeper area to some extent. This phenomenon produces some vertical mixing back to produce B-lines. Studies indicate that a large number of B-line shadows were signs of pulmonary interstitial syndrome, and the number increased with the decrease of air content and the increase of lung tissue density [10].

When the lung gas content is further reduced, the lung tissue becomes consolidated and the acoustic image can be regarded as a solid tissue similar to the echo of the liver and spleen. Pulmonary consolidations a progressive outcome that can be caused by pulmonary embolism, intrapulmonary cancer metastasis, compression or obstructive atresia, and pulmonary contusion. Consolidation of marginal tissue, the presence of air and fluid, or vascular fusion may further suggest consolidation of the lung. Based on a variety of settings and clinical conditions, ultrasound diagnosis of the lungs is now known as "bedside ultrasound," which will be evaluated along with clinical perspective to make the test most effective and accurate for the diagnosis of specific lung diseases.

However, ultrasound of the lungs has some limitations. One limitation of pulmonary ultrasound examination is the dependence of the patient, which may present challenges in certain situations, including in obese patients due to increased adiposity, patients with subcutaneous emphysema, or those with chest dressing coverage, all which may hinder the transmission of ultrasound. Ultrasound of the lungs does not rule out the diagnosis of lung abnormalities that do not involve the pleura. In particular, some tumors are difficult to detect when they are located in the center of the lung. Alveolar interstitial syndrome sometimes also occupies subpleural space, and focal alveolar interstitial syndrome can sometimes serve as an external warning of internal pathological states, such as perifocal interstitial edema caused by inflammation or impaired lymphatic drainage.

In conclusion, ultrasound has several benefits as a lung imaging diagnostic techniques, including being noninvasive, simple to operate, accurate, and reliable. Ease of repeat ultrasound scans also facilitates the advantage of dynamic observation. The portability of ultrasound also reduces the transport and movement of critically ill patients. It also minimizes radiation exposure. Ultrasound can provide immediate information on lung morphology, for a variety of lung diseases allowing for early diagnosis, dynamic assessment, and follow-up which has important clinical significance. As a beneficial supplement to X-ray and CT examination, pulmonary ultrasound is an important direction for future development and has a good prospects for further application. With the promotion of visualization and non-invasive medicine today, it is believed that with the continuous development of ultrasound technology, which will have a broader potential in clinical and anesthesiology fields, and it is worth further promotion.

\section{Acknowledgement}

We wish to thank Ms. Joyce Y. Liu for editing our manuscript.

\section{Conflict of Interest}

The authors have no conflict of interest to declare. 


\section{References}

[1] World Health Organization. Coronavirus disease (COVID-19) outbreak. 12 January, 2020. Available from: https://www.who.int/ emergencies/diseases/novel-coronavirus-2019.

[2] Hong H, Wang Y, Chung HT, Chen CJ. Clinical characteristics of novel coronavirus disease 2019 (COVID-19) in newborns, infants and children. Pediatr Neonatol 2020; 61: 131-132.

[3] Zhou F, Yu T, Du R, Fan G, Liu Y, Liu Z, et al. Clinical course and risk factors for mortality of adult inpatients with COVID-19 in Wuhan, China: a retrospective cohort study. Lancet 2020; 395: 10541062.

[4] Picano E, Scali MC, Ciampi Q, Lichtenstein D. Lung ultrasound for the cardiologist. JACC Cardiovasc Imaging 2018; 11: 1692-1705.

[5] Soldati G, Smargiassi A, Inchingolo R, Buonsenso D, Perrone T, Briganti DF, et al. Proposal for international standardization of the use of lung ultrasound for patients with COVID-19: a simple, quantitative, reproducible method. J Ultrasound Med. 2020 Mar 30. DOI: $10.1002 /$ jum.15285.

[6] Lichtenstein DA, Malbrain MLNG. Lung ultrasound in the critically ill (LUCI): A translational discipline. Anaesthesiol Intensive Ther
2017; 49: 430-436.

[7] Shrestha GS, Weeratunga D, Baker K. Point-of-Care Lung Ultrasound in Critically ill Patients. Rev Recent Clin Trials 2018; 13(1): 15-26.

[8] Smith MJ, Hayward SA, Innes SM, Miller A. Point-of-care lung ultrasound in patients with COVID-19 - a narrative review. Anaesthesia. 2020 Apr 10. DOI: 10.1111/anae.15082.

[9] General Office of National Health Commission, General Office of National Administration of Traditional Chinese Medicine. Diagnostic and treatment protocol for Novel Coronavirus Pneumonia (Trial version 7). Available from: http://www.nhc.gov.cn/yzygj/s7653p/202 003/46c9294a7dfe4cef80dc7f5912eb1989.shtml.

[10] Lichtenstein DA. BLUE-protocol and FALLS-protocol: two applications of lung ultrasound in the critically ill. Chest 2015; 147 : 1659-1670.

[11] Ferro F, Delle Sedie A. The use of ultrasound for assessing interstitial lung involvement in connective tissue diseases. Clin Exp Rheumatol 2018; 36 Suppl 114: 165-170.

[12] Soldati G, Demi M. The use of lung ultrasound images for the differential diagnosis of pulmonary and cardiac interstitial pathology. J Ultrasound 2017; 20: 91-96. 